\title{
COMPLETELY MONOTONIC FUNCTION ASSOCIATED WITH THE GAMMA FUNCTIONS AND PROOF OF WALLIS' INEQUALITY
}

\author{
CHAO-PING CHEN AND FENG QI
}

Abstract. We prove: (i) A logarithmically completely monotonic function is completely monotonic. (ii) For $x>0$ and $n=0,1,2, \ldots$, then

$$
(-1)^{n}\left(\ln \frac{x \Gamma(x)}{\sqrt{x+1 / 4} \Gamma(x+1 / 2)}\right)^{(n)}>0 .
$$

(iii) For all natural numbers $n$, then

$$
\frac{1}{\sqrt{\pi(n+4 / \pi-1)}} \leq \frac{(2 n-1) ! !}{(2 n) ! !}<\frac{1}{\sqrt{\pi(n+1 / 4)}} .
$$

The constants $\frac{4}{\pi}-1$ and $\frac{1}{4}$ are the best possible.

A function $f$ is said to be completely monotonic on an interval $I$, if $f$ has derivatives of all orders on $I$ and satisfies

$$
(-1)^{n} f^{(n)}(x) \geq 0 \quad(x \in I ; n=0,1,2, \ldots) .
$$

If the inequality (1) is strict, then $f$ is said to be strictly completely monotonic on $I$. Completely monotonic functions have remarkable applications in different branches. For instance, they play a role in potential theory [2], probability theory $[4,7,9]$, physics [6], numerical and asymptotic analysis [8, 14], and combinatorics [1]. A detailed collection of the most important properties of completely monotonic functions can be found in [13, Chapter IV], and in an abstract in [3].

A positive function $f$ is said to be logarithmically completely monotonic on an interval $I$ if its logarithm $\ln f$ satisfies

$$
(-1)^{n}[\ln f(x)]^{(n)} \geq 0 \quad(x \in I ; n=1,2, \ldots) .
$$

Received and revised February 17, 2003.

2000 Mathematics Subject Classification. Primary 33B15, 26A48, 26D07, Secondary 30E20.

Key words and phrases. Gamma function, Complete monotonicity, Wallis' inequality.

The authors were supported in part by NNSF (\#10001016) of China, SF for the Prominent Youth of Henan Province (\#0112000200), SF of Henan Innovation Talents at Universities, Doctor Fund of Jiaozuo Institute of Technology, China. 
If the inequality (2) is strict, then $f$ is said to be strictly logarithmically completely monotonic, see [10].

The gamma function

$$
\Gamma(z)=\int_{0}^{\infty} t^{z-1} e^{-t} d t \quad(\operatorname{Re} z>0)
$$

is one of the most important function in analysis and its applications. The history and the development of this function are described in detail in [5]. The logarithm of the gamma function can be expressed [11, p.152] as

$$
\ln \Gamma(z)=\int_{0}^{\infty}\left[e^{-t}(z-1)+\frac{e^{-z t}-e^{-t}}{1-e^{-t}}\right] \frac{d t}{t} \quad(\operatorname{Re} z>0) .
$$

In this paper, we obtain the following results.

Theorem 1. If the function $\phi$ defined on an interval $I$ is (strictly) completely monotonic, then $\exp \phi$ is also (strictly) completely monotonic on I.

Proof. Since $\phi$ is completely monotonic on $I$, we have

$$
(-1)^{k} \phi^{(k)}(x) \geq 0 \quad(x \in I ; k=0,1,2, \ldots) .
$$

It is clear that $\exp \phi(x) \geq 1,[\exp \phi(x)]^{\prime}=\phi^{\prime}(x) \exp \phi(x) \leq 0$ and $[\exp \phi(x)]^{\prime \prime}=\left\{\phi^{\prime \prime}(x)+\right.$ $\left.\left[\phi^{\prime}(x)\right]^{2}\right\} \exp \phi(x) \geq 0$, that is, for $x \in I$ and $k=0,1,2$, we have

$$
(-1)^{k}[\exp \phi(x)]^{(k)} \geq 0
$$

Suppose (4) holds for all nonnegative integers $k \leq n$. By Leibnitz's formula, we have

$$
\begin{aligned}
& (-1)^{n+1}[\exp \phi(x)]^{(n+1)}=(-1)^{n+1}\left\{[\exp \phi(x)]^{\prime}\right\}^{(n)} \\
= & (-1)^{n+1}\left[\phi^{\prime}(x) \exp \phi(x)\right]^{(n)}=(-1)^{n+1} \sum_{i=0}^{n}\left(\begin{array}{c}
n \\
i
\end{array}\right) \phi^{(i+1)}(x)[\exp \phi(x)]^{(n-i)} \\
= & \sum_{i=0}^{n}\left(\begin{array}{c}
n \\
i
\end{array}\right)\left[(-1)^{i+1} \phi^{(i+1)}(x)\right]\left\{(-1)^{n-i}[\exp \phi(x)]^{(n-i)}\right\} \geq 0 .
\end{aligned}
$$

By induction, it is proved that the function $\exp \phi$ is completely monotonic on $I$.

In the proof of Theorem 1, we see that if the function $\phi$ is strictly completely monotonic on $I$, then $\exp \phi$ is also strictly completely monotonic on $I$. The proof is complete.

Theorem 2. For $x>0$ and $n=0,1,2, \ldots$, then

$$
(-1)^{n}\left(\ln \frac{x \Gamma(x)}{\sqrt{x+1 / 4} \Gamma(x+1 / 2)}\right)^{(n)}>0 .
$$


Proof. Using (3) and the representation

$$
\ln x=\int_{0}^{\infty} \frac{e^{-t}-e^{-x t}}{t} d t \quad(x>0),
$$

we conclude that

$$
\begin{aligned}
& \ln \frac{x \Gamma(x)}{\sqrt{x+1 / 4} \Gamma(x+1 / 2)} \\
= & \ln \Gamma(x)-\ln \Gamma(x+1 / 2)+\ln x-\frac{1}{2} \ln (x+1 / 4) \\
= & \frac{1}{2} \int_{0}^{\infty} \frac{e^{t / 4}+e^{-t / 4}-2}{1+e^{t / 2}} \cdot \frac{e^{-x t}}{t} d t>0 \quad(x>0) .
\end{aligned}
$$

From (5) we conclude that

$$
(-1)^{n}\left(\ln \frac{x \Gamma(x)}{\sqrt{x+1 / 4} \Gamma(x+1 / 2)}\right)^{(n)}=\frac{1}{2} \int_{0}^{\infty} \frac{e^{t / 4}+e^{-t / 4}-2}{1+e^{t / 2}} \cdot \frac{e^{-x t}}{t^{1-n}} d t>0
$$

for $x>0$ and $n=0,1,2 \ldots$. The proof is complete.

Remark. From (5) we get

$$
\frac{x \Gamma(x)}{\sqrt{x+1 / 4} \Gamma(x+1 / 2)}>1 \quad(x>0) .
$$

In fact, using the asymptotic expansion (see [8])

$$
x^{b-a} \frac{\Gamma(x+a)}{\Gamma(x+b)}=1+\frac{(a-b)(a+b-1)}{2 x}+O\left(x^{-2}\right) \quad(x \rightarrow \infty),
$$

we conclude that

$$
\lim _{x \rightarrow \infty} \frac{x \Gamma(x)}{\sqrt{x+1 / 4} \Gamma(x+1 / 2)}=1 .
$$

By Theorem 1, the function $f(x)=\frac{x \Gamma(x)}{\sqrt{x+1 / 4} \Gamma(x+1 / 2)}-1$ is strictly completely monotonic on $(0, \infty)$.

As an application of (6), we prove the following Wallis' inequality [12].

Theorem 3. For all natural numbers $n$, then

$$
\frac{1}{\sqrt{\pi(n+4 / \pi-1)}} \leq \frac{(2 n-1) ! !}{(2 n) ! !}<\frac{1}{\sqrt{\pi(n+1 / 4)}},
$$

where $(2 n) ! !=\prod_{k=1}^{n}(2 k)$ and $(2 n-1) ! !=\prod_{k=1}^{n}(2 k-1)$. The constants $\frac{4}{\pi}-1$ and $\frac{1}{4}$ are the best possible. 
Proof. First, we show that the sequence

$$
Q_{n}=\left[\frac{\Gamma(n+1)}{\Gamma\left(n+\frac{1}{2}\right)}\right]^{2}-n \quad(n=1,2, \ldots)
$$

is strictly decreasing. It is sufficient to show that $Q_{n+1}<Q_{n}$, which is equivalent to

$$
\frac{\Gamma(n+1)}{\Gamma\left(n+\frac{1}{2}\right)}<\frac{2 n+1}{\sqrt{4 n+3}}
$$

Take in (6) $x=n+\frac{1}{2},(9)$ holds clearly.

Now, we prove (8). Since

$$
\Gamma(n+1)=n !, \quad \Gamma\left(n+\frac{1}{2}\right)=\frac{(2 n-1) ! !}{2^{n}} \sqrt{\pi}, \quad 2^{n} n !=(2 n) ! !,
$$

the inequality (8) is equivalent to

$$
\frac{1}{4}<Q_{n}=\left[\frac{\Gamma(n+1)}{\Gamma\left(n+\frac{1}{2}\right)}\right]^{2}-n \leq \frac{4}{\pi}-1 .
$$

From the monotonicity of the sequence $Q_{n}$, it follows that

$$
\lim _{n \rightarrow \infty} Q_{n}<Q_{n} \leq Q_{1}=\frac{4}{\pi}-1 .
$$

Using the asymptotic expansion (7) we conclude from

$$
Q_{n}=n\left[n^{-\frac{1}{2}} \frac{\Gamma(n+1)}{\Gamma\left(n+\frac{1}{2}\right)}-1\right]\left[n^{-\frac{1}{2}} \frac{\Gamma(n+1)}{\Gamma\left(n+\frac{1}{2}\right)}+1\right]
$$

that

$$
\lim _{n \rightarrow \infty} Q_{n}=\frac{1}{4}
$$

Thus, the inequality (8) follows. The proof is complete.

\section{References}

[1] K. Ball, Completely monotonic rational functions and Hall's marriage theorem, J. Comb. Th., Ser. B 61 (1994), 118-124.

[2] C. Berg and G. Forst, Potential Theory on Locally Compact Abelian Groups, Ergebnisse der Math. 87, Springer, Berlin, 1975.

[3] C. Berg, J. P. R. Christensen and P. Ressel, Harmonic Analysis on Semigroups. Theory of Positive Definite and related Functions, Graduate Texts in Mathematics 100, Springer, Berlin-Heidelberg-New York, 1984.

[4] L. Bondesson, Generalized Gamma Convolutions and related Classes of Distributions and Densities, Lecture Notes in Statistics 76, Springer, New York, 1992. 
[5] P. J. Davis, Leonhard Euler's integral: A historical profile of the gamma function, Amer. Math. monthly 66 (1959), 849-869.

[6] W. A. Day, On monotonicity of the relaxation functions of viscoelastic meterial, Proc. Cambridge Philos. Soc. 67 (1970), 503-508.

[7] W. Feller, An Introduction to Probability Theory and its Applications, Vol. 2, Wiley, New York, 1966.

[8] C. L. Frenzen, Error bounds for asymptotic expansions of the ratio of two gamma functions, SIAM J. Math. Anal. 18 (1987), 890-896.

[9] C. H. Kimberling, A probabilistic interpretation of complete monotonicity, Aequat. Math. 10 (1974), 152-164.

[10] F. Qi and S.-L. Xu, The function $\left(b^{x}-a^{x}\right) / x$ : Inequalities and properties, Proc. Amer. Math. Soc. 126 (1998), 3355-3359.

[11] Tan Lin, Reading Notes on Gamma Function, Zhejiang university Press, Hangzhou City, China, 1997. (in Chinese)

[12] G. N. Watson, A note on gamma functions, Proc. Edinburgh Math. Soc. 11 1958/1959 Edinburgh Math. Notes No. 42 (misprinted 41) (1959), 7-9.

[13] D. V. Widder, The Laplace Transform, Princeton Univ. Press, Princeton, NJ, 1941.

[14] J. Wimp, Sequence Transformations and their Applications, Academic Press, Nex York, 1981.

Department of Applied Mathematics and Informatics, Jiaozuo Institute of Technology, \#142, Mid-Jiefang Road, Jiaozuo City, Henan 454000, China.

E-mail: chenchaoping@sohu.com

Department of Applied Mathematics and Informatics, Jiaozuo Institute of Technology, \#142, Mid-Jiefang Road, Jiaozuo City, Henan 454000, China.

E-mail: qifeng@jzit.edu.cn or qifeng618@jzit.edu.cn 